\title{
Influence of manual dominance on the unimanual and bimanual activities of post-stroke patients treated with constraint-induced therapy
}

\author{
NATHALIA TRASMONTE DA SILVA ${ }^{1} \mid$ JACKELINE HARUMI YONOBI ${ }^{1} \mid$ RAFAEL ERAS-GARCIA ${ }^{1} \mid$ JULIANA LEME \\ GOMES ${ }^{2} \mid$ CLARISSA BARROS DE OLIVEIRA ${ }^{2,3 \mid}$ GABRIELA DA SILVA MATUTI ${ }^{2}$
}

1 Department of Occupational Therapy at Associação de Assistência à Criança Deficiente (AACD - Ibirapuera), São Paulo, Brazil

2 Department of Physiotherapy at Associação de Assistência à Criança Deficiente (AACD - Ibirapuera), São Paulo, Brazil;

${ }^{3}$ Supervisor at department of Physiotherapy at Associação de Assistência à Criança Deficiente (AACD - Ibirapuera), São Paulo, Brazil.

Correspondence to: Nathalia Trasmonte da Silva. Av. Dona Ana Costa, 95, Vila Mathias, City: Santos/SP - Brazil - 11060-001.

email: nathalia.trasmonte@gmail.com

\section{AT A GLANCE \\ Both groups showed similar improvement in the amount and quality of use of the affected upper limb, suggesting that CIT is effective independent of previous manual dominance. This indicates that therapists do not have to be concerned of previous manual dominance to list subjects to CIT rehabilitation, as both concordance and non-dominant patients may have the same potential for functional improvement.

$\begin{array}{ll}\text { ABBREVIATIONS } \\ \text { AOU } & \text { amount of use } \\ \text { CIT } & \text { Constraint-induced therapy } \\ \text { CG } & \text { concordance group } \\ \text { MAL } & \text { Motor Activity Log } \\ \text { MMSE } & \text { Mini-Mental Statue Exam } \\ \text { NDG } & \text { non-dominant group } \\ \text { QOM } & \text { quality of movement } \\ \text { UL } & \text { upper limb } \\ \text { WMFT } & \text { Wolf Motor Function Test }\end{array}$

PUBLICATION DATA

Received 21 Dec 2016

Accepted 26 Ago 2017

Published 02 Set 2017

\begin{abstract}
BACKGROUND: Constraint-induced therapy (CIT) has long-term beneficial effects. However, there is evidence that the recovery of the functional movements of the affected hand can be influenced by hand dominance prior to the brain injury and that this aspect may influence the maintenance of the CIT results.

AIM: To investigate the influence of previous hand dominance in post-stroke hemiparetic patients on immediate CIT results and result maintenance in unimanual and bimanual activities for at least 12 months.

METHOD: This was an observational study that used medical records of patients who performed the CIT protocol between 2009 and 2014. Thirty-four patients were included and divided into two groups: concordance group ( $\mathrm{n}=18$; age: 56(13); sex: 11 male) and non-dominant group ( $n=16$; age: 57(13); sex: 9 male). Two scales were used: the Motor Activity Log (MAL), and the Wolf Motor Function Test (WMFT).

RESULTS: MAL and WMFT scales showed significant improvement comparing pre- to post-treatment. However, there was no statistical difference between the groups at any follow-up on both scales.

CONCLUSION: There was significant improvement and maintenance in both the quality and the amount of use in the more affected upper limb, but the previous manual dominance was not a relevant factor.
\end{abstract}

KEYWORDS: Constraint-induced therapy| Hand dominance| Unimanual and bimanual activities| Stroke| Neurological rehabilitation.

\section{INTRODUCTION}

Stroke that does not lead to death can cause impairments and changes that vary according to the area and extent of the injury. ${ }^{1}$ The impairments can be sensory, motor and/or cognitive and interfere with the performance, functional motor capacity and quality of life of the individual. Hemiparesis or hemiplegia is an important stroke motor sequel that consists of a physical state composed of weakness or unilateral paralysis, respectively. ${ }^{2}$ One notable deficit presented by the hemiparetic individual is the greater involvement of the upper limb (UL) contralateral to the brain injury. ${ }^{3}$ Consequently, the non-affected upper limb starts to perform most of the functional tasks and causes a higher disuse of the paretic UL. ${ }^{3}$

Constraint-induced therapy $(\mathrm{CIT})$ is an important intervention to improve the upper limb 
Brazilian Journal of Motor Behavior

motor functions and is recognized as the gold standard for rehabilitating patients with stroke impairments. ${ }^{4}$ The CIT components include immobilization of the unaffected UL, specific and intensive training tasks for the affected UL and the behavioral methods package, which serves as the technical differential. So, CIT improves dexterity, the perception of arm and hand use, the quality of movement of the affected UL, and the quality of life of patients with stroke impairments. ${ }^{4}$

CIT generates long-term effects and its benefits can last for at least two years after the end of the protocol. ${ }^{5}$ However, as the left and right hemispheres of the brain are specialized for specific functions ${ }^{6}$, the patient's hand dominance should be considered when evaluating the maintenance of the results. ${ }^{3}$ There are indications that the recovery of the various functional movements levels of the affected hand is based on hand dominance prior to the injury. ${ }^{3}$ Previous manual dominance has been mentioned as an important factor in UL motor skill performance, presenting better speed, accuracy, coordination and strength than the UL found on the non-dominant side. ${ }^{6}$

Predictors that have potential to influence the CIT results are the side of stroke, the time since stroke, age, sex, and ambulatory status. ${ }^{7}$ Lima et al. $(2014)^{8}$ evaluated the influence of previous manual dominance on the $\mathrm{CIT}$ protocol and although in the immediate results there was no difference between groups, at the 3-month follow-up measure only the individuals whose paretic upper limb was the dominant maintained the results. Langan and van Donkelaar $(2007)^{3}$ suggest that prior manual dominance may have higher influence when the post-stroke functional deficit is higher, but this has not been proven. Although dominance is discussed in these aspects, there is no scientific data related to the interference of previous manual dominance in performing unimanual and bimanual activities, considering 1-year follow-up beyond the immediate results.

According to Hayashi and Nozaki $(2016)^{9}$, the training of a specific task in a unimanual manner is beneficial because once the task is first trained in this way, the same action can be practiced bimanually more easily thereafter. The authors demonstrated that the learning of new movements is partially transferred to the moving arm when the contralateral arm is restricted (immovable).

Thus, our hypothesis is that previous manual dominance influences the immediate and longterm outcomes of CIT in unimanual and bimanual activities in post stroke patients. To test this hypothesis, we analyzed the influence of hand dominance prior to injury on the immediate CIT results in post-stroke hemiparetic patients as well as the result maintenance for at least 12 months for both unimanual and bimanual activities.

\section{METHODS}

From 2009 to 2014, the CIT protocol was performed on 119 patients with a stroke diagnosis and more than three months of injury. To receive the therapy, the patients had to meet the motor and cognitive criteria established by the original CIT protocol. All patients underwent the protocol currently recommended by the University of Alabama at Birmingham (i.e., 10 consecutive days for 3 hours/day)..$^{10}$ All procedures were approved by the Committee of Ethics and Research from the institution, according to the Approval Number 1179052.

The motor criteria were as follows: $45^{\circ}$ shoulder flexion and/or abduction, $20^{\circ}$ elbow extension, $10^{\circ}$ wrist extension, at least $10^{\circ}$ of metacarpophalangeal and interphalangeal extension of at least two fingers and $10^{\circ}$ of thumb abduction and/or extension. The functional levels were classified based on the active range of motion of the shoulder, elbow, wrist, fingers and thumb of the affected UL. The score of CIT's motor degree scale is gradual and ranges from two to five points, in 
Brazilian Journal of Motor Behavior

which a score of two corresponds to a mild impairment and five corresponds to a serious impairment. ${ }^{11,12}$

The cognitive criteria included the individual being able to follow simple commands, understand the technical objectives, commit to participate and answer the protocol scales appropriately. Although there is no consensus in the literature regarding the most appropriate method to evaluate these criteria, the majority of researchers use the Mini-Mental Statue Exam (MMSE), whose score is often adopted as a cutoff greater than or equal to 24 .

For this study, we selected 34 patients with a functional level of 2 (mild or moderate impairment) on the functional ability scale ${ }^{11,12}$ and a MMSE score greater than 24 points who answered all necessary scales prior to and after treatment and on each of the 10 days of therapy. Another 85 patients were excluded because they had missing or illegible data on their medical records or because they did not attend the follow-ups at 1, 3,6 and/or 12 months.

According to literature, when the affected hand is the dominant hand, this is called "concordance". ${ }^{13}$ So, this terminology will be used here to refer to this population. Although literature recognize the Edinburgh Inventory(14) the standard to determine previous manual dominance, the manual dominance was determined by the hand that the patient used to write before the injury.(8) Thereby, the selected patients for the study were divided into two groups - CG (concordance group): $n=18$, dominant hand affected by stroke and NDG (non-dominant group): $n=16$, non-dominant hand affected by stroke. The baseline demographic and clinical characteristics for each group are presented on Table 1.

\begin{tabular}{ccc}
\hline & \multicolumn{2}{c}{ Patients $(\mathbf{n = 3 4 )}$} \\
& Concordance Group ( $\mathbf{n = 1 8 )}$ & Non-dominant Group $(\mathbf{n = 1 6})$ \\
\hline Sex (male) & 11 & 9 \\
years) & $56(13)$ & $57(13)$ \\
Functional Level & $100 \%$ mild & $88 \%$ mild \\
Etiology (ischemic) & $72 \%$ & $62 \%$ \\
Average and SD of time from & 46 months (38.86) & 42.6 months $(42.35)$ \\
injury & & 28 points $(2)$ \\
Average and SD of MMSE & 27 points (3) & \\
score & &
\end{tabular}

Table 1: Baseline demographic and clinical characteristics of each group.SD: standard deviation

The CIT has its own evaluation scales, the Motor Activity Log (MAL) and the Wolf Motor Function Test (WMFT), which have both been validated for application in Brazil. 5,15 The main evaluation instrument to assess and monitor the CIT results is the MAL, which allows evaluators to assess self-reported paretic arm level of activity outside the therapeutic environment. The MAL scores reproduce the competence of the individual in performing the daily living activities ${ }^{16}$ and the scores are given by the patient. ${ }^{17,18}$

The MAL is a structured interview applied at pretreatment, post-treatment and on the

\begin{tabular}{|l|l|l|l}
\hline 2017 & VOL. 11 & N.1
\end{tabular}


Brazilian Journal of Motor Behavior

subsequent follow-up visits. The scale assesses the patient's perception about the real use of the affected upper limb through thirty common functional tasks in the patient's daily life and is divided into two subscales: amount of use (AOU) and quality of movement (QOM). The score ranges from zero to five points, that is, zero indicates no use of the affected arm during the activity and five represents the use of the affected arm as often/well as before the injury. $(17,18)$ For the analysis, the tasks were divided according to their characteristics [unimanual $(n=24)$ or bimanual $(n=6)$ ] to establish criteria to quantify differences between the concordance and non-dominant groups. We also identified and analyzed the AOU subscale items considering the pre-treatment, post-treatment and one-year follow-up examinations to identify whether there were any specific tasks in which the patient lost quantitative long-term use. Use of the affected upper limb below $50 \%$ on the AOU subscale (a score of 3 for AOU in the upper limb) was set as a risk for asymmetry reoccurrence. 5,19

The WMFT is a scale that measures the upper extremity movement ability to execute fifteen unimanual tasks, which include isolated movements and functional activities, by timing how long it takes the patient to perform each task. The final score is determined from the arithmetic mean.(15) According to Wolf et al (2001) ${ }^{20}$, the activities are performed in gradual complexity, advancing from axial to distal joint, testing the total speed and quality of movement, and require simple tools and minimal training to be applied.

The two scales (MAL and WFMT) were used in this study to evaluate the immediate effects of the technique, but only the MAL was used to analyze the maintenance of the results at the followup examination ${ }^{5}$ because the WMFT was performed only before and after treatment according to the protocol. ${ }^{12}$

Data including gender, age, period of injury and rehabilitation prior to the CIT were collected to characterize the samples. The sample calculation was made considering the study of Lima et al. (2014)(8), which used a similar method. We used the $G^{*}$ Power software, repeated-measures ANOVA, a 0.05, $\beta 0.80,2$ groups, 2 measurements and an effect size (difference between groups) of the endpoint scales (AOU and QOM - Motor Activity Log) which were $f=2.2$ and $f=0.6$ respectively. These values gave a sample size of at least 24 patients to a $\beta$ power of 0.80 .

Data normality was tested and Two-Way ANOVA for repeated measures test (factor: groups and time of evaluation) was used for AOU and QOM for unimanual and bimanual activities and also for WMFT analysis. For between groups comparison at each time point (pre-treatment, posttreatment and annual) for MAL unimanual and bimanual separated activities, an unpaired t test was employed. Cohen's $d$ was used for effect size determination and the values are presented at the figures legend. The statistical analysis was performed on the SPSS V17, Minitab 16 software with a statistical significance level of $p<0.05$.

\section{RESULTS}

There was a significant difference for the factor time of evaluation for AOU and QOM for both unimanual and bimanual activities. CIT improved the AOU of the affected upper limb in uni$(F 5,160=139, p<0.0001)$ and bi-manual $(F 5,160=54.77, p<0.0001)$ tasks immediately after the treatment (pos-treatment) and throughout the follow-up. Similarly, QOM was also improved by CIT in uni- $(F 5,160=104.4, p<0.0001)$ as well as bimanual $(F 5,160=94.59, p<0.0001)$ tasks. No effect for the factor groups was observed (Figures 1 and 2), indicating that there was no difference due the previous manual dominance.

\begin{tabular}{l|l|l|l|l}
\hline Silva et al. & 2017 & VOL. 11 & N.1 \\
\hline
\end{tabular}




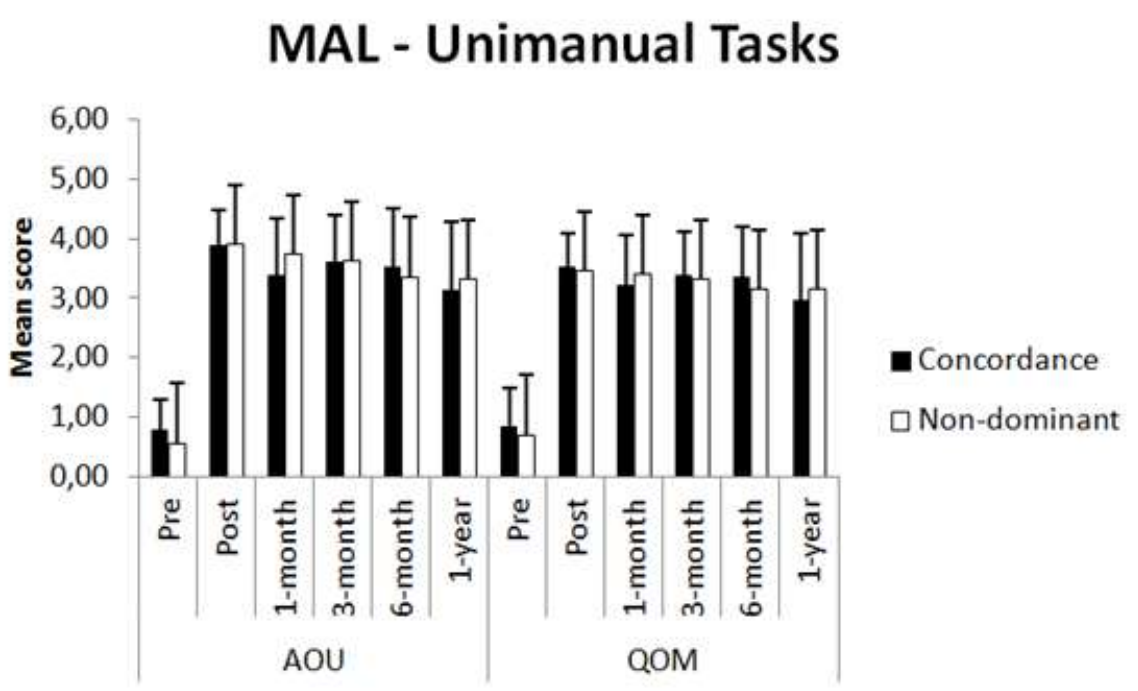

Figure 1: Analysis of amount of use (AOU) and quality of movement (QOM) of the affected upper limb in unimanual activities at each time point according to the MAL scale. Concordance group, $n=18$; non-dominant group, $n=16$. Results are presented as mean + standard deviation. *effect of time of evaluation, $p<0.0001$. For AOU, Cohen's $d$ values (pre compared to post, 1-month, 3-month, 6-month and 1-year) were 5.66, 3.37, 5.14, 3.55 and 2.64 for the Concordance group and 5.66, 4.91, 4.50, 3.38 and 2.86 for the Non-dominant group. For QOM, Cohen's d values were 4.37, 3.15, $3.62,3.27$ and 2.34 for the Concordance group and 3.43, 4.41, 3.30, 2.84 and 2.53 for the Non-dominant group.

\section{MAL - Bimanual tasks}

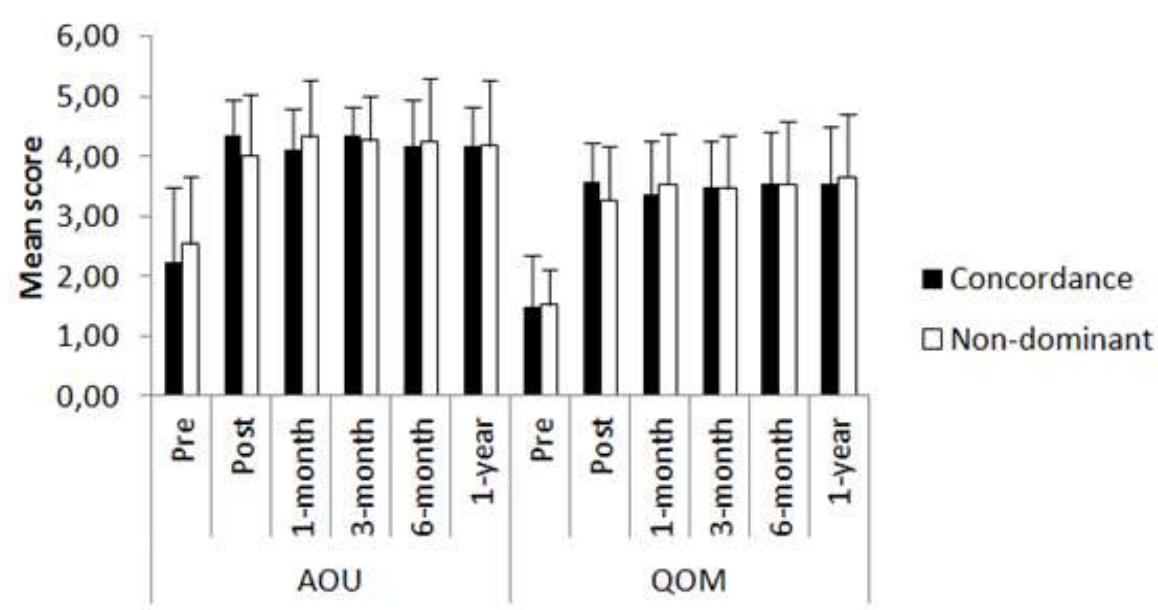

Figure 2: Analysis of amount of use (AOU) and quality of movement (QOM) of the affected upper limb in bi-manual activities at each time point according to the MAL scale. Concordance group, $n=18$; non-dominant group, $n=16$. Results are presented as mean + standard deviation. *effect of time of evaluation, $p<0.0001$. For AOU, Cohen's $d$ values (pre compared to post, 1-month, 3-month, 6-month and 1-year) were 2.16, 1.84, 2.19, 1.87 and 1.91 for the Concordance group and $1.41,1.75,1.85,1.61$ and 1.49 for the Non-dominant group. For QOM, Cohen's d values were 2.69, 2.14, 2.40, 2.39 and 2.24 for the Concordance group and 2.27, 2.77, 2.52, 2.33 and 2.51 for the Non-dominant group.

When the MAL activities were divided into unimanual $(n=24)$ and bimanual $(n=6)$ tasks, we observed only three tasks with significant results after comparison between groups. During pretreatment, task number 4 (pick up the phone, $p=0.025$ ) and task number 26 (use a fork or spoon for 
eating, $p=0.025$ ) showed significant results, with the NDG demonstrating less use of the affected upper limb. During post-treatment, only task number 28 (pick up a cup by the wrist, $p=0.042$ ) was significantly different between the groups, with the CG demonstrating less use of the affected upper limb. For all tasks, there were no significant differences between groups during the follow-up examinations for at least until 1 year after the end of the protocol.

CIT increased significantly the movement execution speed, as determined by the WMFT (effect of the factor time of evaluation, $F 1,32=20.25, p<0.001$ ). Again, no difference for the factor groups was found (Figure 3).

\section{WMFT}

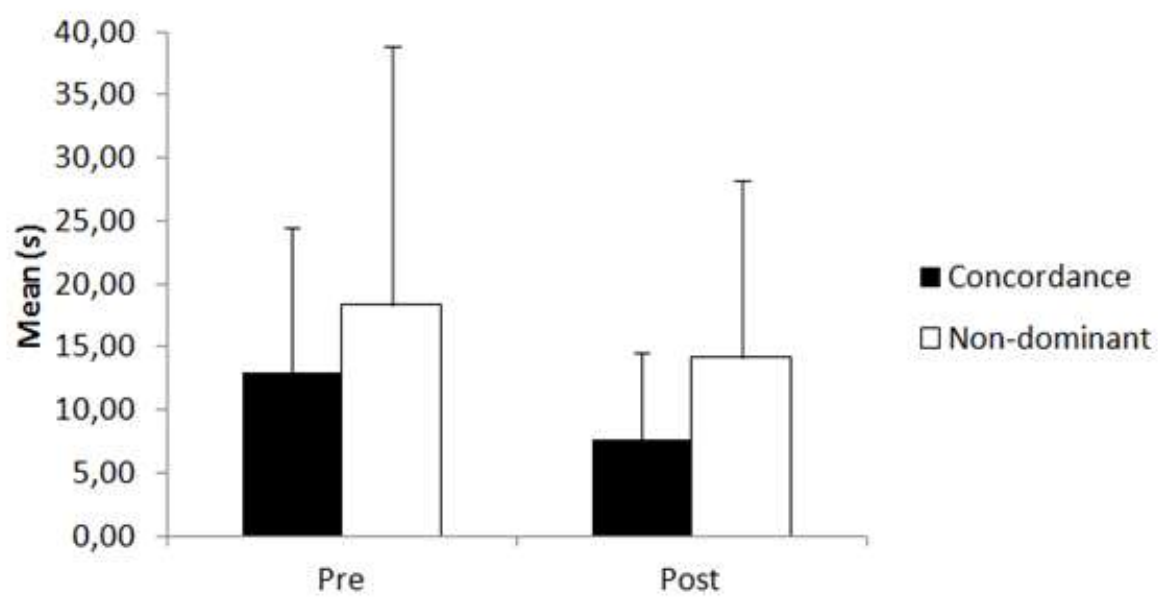

Figure 3: Pre- and post-treatment WMFT assessment in concordance and non-dominant groups. Concordance group, $\mathrm{n}=18$; non-dominant group, $\mathrm{n}=16$. Results are presented as mean + standard deviation. *effect of time of evaluation, $p<0.0001$. WMFT: Wolf Motor Functional Test. The Cohen's d values (pre compared to post) was 0.56 for the Concordance group and 0.22 for the Non-dominant group.

\section{DISCUSSION}

Patients with stroke impairments may experience an increase in the use of the less affected $U L$ and consequently a decrease in the use of the affected UL when performing their daily living activities, which characterizes "learned nonuse". ${ }^{21}$ When patients fail in the attempt to accomplish a certain motor activity, behavioral suppression occurs for the execution of that movement. This, in turn, leads the patient to significantly reduce the functional use of the affected UL when combined with brain damage and a decrease in cortical representation areas. ${ }^{12}$ This non-use also weakens certain muscle groups and decreases the upper limb's ability to perform both types of activities (unimanual and bimanual). ${ }^{8}$

Given the previously mentioned factors, it is extremely important to treat these deficits. According to Langan and Van Donkelaar (2008)3 , CIT has short- and long-term benefits. However, it is clear that the majority of the population executes daily living activities more efficiently with one of their upper limbs, which reveals dominance. 
Lima et al. (2014) ${ }^{8}$ compared the immediate CIT results and their maintenance for up to 3 months in patients with mild to moderate chronic stroke impairments considering their previous dominance. Similar to our results, acquisition of abilities (the difference between pre-and posttreatment) was not affected by previous manual dominance. However, regarding the maintenance of the results, they concluded that for unimanual activities the dominance was a relevant factor at 3month follow-up, whereas in this study both CG and NDG group were able to maintain their previous gains for up to 1 year in unimanual and bimanual activities. This contrast of results may be due to the fact that Lima et al. (2014) ${ }^{8}$ classified the degree of commitment of their patients by the FuglMeyer scale, which despite being widely used for post-stroke patients, can generate a distinct classification of the CIT's motor degree scale ${ }^{12}$, allowing the inclusion of individuals with different degrees of severity that would not usually be included. In addition, in the study cited, the subjects were submitted to the home-based modified protocol of $\mathrm{CIT}$, while in this study the subjects were submitted to the original and standardized protocol, which may be a factor that will strengthen our results.

There is limited discussion in the literature on the ability of post-stroke individuals to perform unimanual and bimanual activities. Gosser and Rice (2015)22 suggested that the unaffected UL showed continuous movement strategies in patients with stroke impairments and in contrast the affected UL presented discontinuous movement patterns. The authors also suggested that many patients were not able to perform bimanual activities after a stroke, which was notably harmful for their functionality because many daily living activities involved both upper limbs in coordinated movements. Other authors reported that the unaffected UL needed to adapt its movements to the restrictions imposed by the affected UL in these types of tasks. ${ }^{23}$ Thus, Gosser and Rice (2015) ${ }^{22}$ suggested that both unaffected and affected ULs had higher execution speeds in unimanual activities than in bimanual activities because there was no interference from the contralateral limb during the unimanual activities.

The results of our study contradict the affirmations mentioned above because there were no significant differences between the CG and NDG in either the quantity or the quality of UL use or in the speed of movement execution in the unimanual and bimanual activities predetermined by our rating scales. Therefore, we show that the CIT protocol is effective at improving the ability of the affected $\mathrm{UL}$ in both types of tasks independent of previous dominance. In a study administering the 6-hour/day CIT protocol for 10 days, Fritz et al. (2006) ${ }^{7}$ found that previous manual dominance was not a predictor of outcomes in the posttreatment and up to 6-months of follow-up. Although the authors had not separating the activities into unimannual and bimanual, they presented similar findings to those presented here.

Moreover, as mentioned previously, learned nonuse is a serious consequence of stroke sequels. In a study that investigated the symmetry of use of the upper extremity in healthy and poststroke patients, the authors concluded that healthy subjects use the hands in a nearly symmetrical way, but post-stroke patients have significant less use of the affected hand. ${ }^{3}$ This fact reinforces the 
Brazilian Journal of Motor Behavior

evidence of learned nonuse, and strengthens the importance of our result as it shows a more symmetrical use of upper limbs after the CIT protocol in post-stroke patients.

The motivational factor and the behavioral package are the two main points of discussion between the results presented here and the current literature24, 25. With respect to motivation, individuals who have neural injury impairments on their dominant side express greater motivation to be able to use the affected hand as they did before. Moreover, because individuals with impairments on their non-dominant side are less motivated to use this side after injury, they have greater difficulty in completing the therapy protocols.(18) Regarding the behavioral package, according to Taub et al. $(2013)^{25}$, it consists of an aggregation of commonly used techniques in the field of behavioral analysis that can be employed as a resource for the treatment of a wide variety of conditions. However, the authors affirm that it has not been used continuously for rehabilitation, which suggests that this is the CIT differential, since CIT is characterized as a primarily behavioral intervention ${ }^{26}$.

Stock et al. (2015) 24 proposed that the motivational factor was linked to the perception of the successful use of the affected UL because the patient felt more motivated to use that UL when improvement was realized. Regarding the transfer package, Taub et al. $(2013)^{25}$ reported that this package helped the patient to become an active subject in his own rehabilitation, which allowed wellestablished tasks to be performed at home and the patient to be immersed in his therapy for a significant amount of the day. These studies give support to the two major points of discussion raised here - motivational factor and behavioral package. This demonstrates the importance of the protocol independent of previous manual dominance, since we didn't find statistical difference between the groups in any of the evaluated moments (pretreatment, post-treatment and the subsequent followup) for both unimanual and bimanual tasks.

Our findings represent preliminary information about this topic and suggest the efficacy of the CIT technique at reversing learned nonuse regardless of manual dominance prior to the injury. In conclusion, based on our data, it is possible to propose that previous manual dominance should not be used as an eligibility criterion for patient participation in CIT protocol, as it did not affect the improvement in the amount and quality of use in both unimanual and bimanual activities, showing that the patients may have the same potential for functional improvement following CIT.

\section{Study Limitations}

There are few limitations of our study including not using a prospective design and the Edinburg Inventory to determine previous manual dominance. Also, we did not use blind evaluators to avoid bias by the evaluators performing the protocol, a control group with conventional therapy and a follow-up after 2 years to continue monitoring the long-term effects. Although the sample size presented here was statistically adequate, it could be interesting future studies to replicate the method with a larger sample. 
1. Lima PN, de Souza CM, da Silva S, Andrade FNC, Braga DM. Fisioterapia Aquática na Transferência do Sentado para Ortostatismo no Paciente com AVC: Relato de Caso. Revista Neurociencias. 2013:251-7.

2. de Andrade LM, Costa MdFM, Caetano JÁ, Soares E, Beserra EP. A problemática do cuidador familiar do portador de acidente vascular cerebral. Revista da Escola de Enfermagem da USP. 2009;43(1):37-43.

3. Langan J, van Donkelaar $P$. The influence of hand dominance on the response to a constraintinduced therapy program following stroke. Neurorehabilitation and neural repair. 2007.

4. Staal J, Hendriks E, Heijmans M, Kiers H, Lutgers-Boomsma A, Rutten G. KNGF Clinical Practice Guideline for Physical Therapy in patients with low back pain. Royal Dutch Society for Physical Therapy (Koninklijk Nederlands Genootschap voor Fysiotherapie, KNGF); 2013. 2014. 5. Pereira ND, Ovando AC, Michaelsen SM, Anjos SMd, Lima RCM, Nascimento LR, et al. Motor Activity Log-Brazil: reliability and relationships with motor impairments in individuals with chronic stroke. Arquivos de neuro-psiquiatria. 2012;70(3):196-201.

6. Provins K. The specificity of motor skill and manual asymmetry: A review of the evidence and its implications. Journal of Motor Behavior. 1997;29(2):183-92.

7. Fritz SL, Light KE, Clifford SN, Patterson TS, Behrman AL, Davis SB. Descriptive characteristics as potential predictors of outcomes following constraint-induced movement therapy for people after stroke. Physical therapy. 2006;86(6):825-32.

8. Lima R, Nascimento LR, Michaelsen SM, Polese JC, Pereira ND, Teixeira-Salmela LF. Influences of hand dominance on the maintenance of benefits after home-based modified constraint-induced movement therapy in individuals with stroke. Brazilian journal of physical therapy. 2014;18(5):435-44.

9. Hayashi T, Nozaki D. Improving a bimanual motor skill through unimanual training. Frontiers in Integrative Neuroscience. 2016;10.

10. Taub E, Uswatte G, Mark VW, Morris DM, Barman J, Bowman MH, et al. Method for Enhancing Real-World Use of a More Affected Arm in Chronic Stroke: Transfer Package of Constraint-Induced Movement Therapy. Stroke. 2013;44:1383-8.

11. Taub E, Crago JE, Burgio LD, Groomes TE, Cook EW, DeLuca SC, et al. An operant approach to rehabilitation medicine: overcoming learned nonuse by shaping. Journal of the experimental analysis of behavior. 1994;61(2):281-93.

12. Wolf SL, Winstein CJ, Miller JP, Taub E, Uswatte G, Morris D, et al. Effect of constraintinduced movement therapy on upper extremity function 3 to 9 months after stroke: the EXCITE randomized clinical trial. Jama. 2006;296(17):2095-104.

13. Nichols-Larsen DS, Clark P, Zeringue A, Greenspan A, Blanton S. Factors influencing stroke survivors' quality of life during subacute recovery. Stroke. 2005;36(7):1480-4.

14. Oldfield RC. The assessment and analysis of handedness: the Edinburgh inventory. Neuropsychologia. 1971;9(1):97-113.

15. Pereira ND, Michaelsen SM, Menezes IS, Ovando AC, Lima RC, Teixeira-Salmela LF. Confiabilidade da versão brasileira do Wolf Motor Function Test em adultos com hemiparesia.

Rev Bras Fisioter. 2011;15(3):257-65.

\begin{tabular}{l|l|l|l|}
\hline Silva et al. & 2017 & VOL. 11 & N.1 \\
\hline
\end{tabular}


16. Garcia JM, Knabben RJ, Pereira ND, Ovando AC. Terapia por Contensão Induzida (TCI) em adolescentes com hemiparesia espástica: relato de caso. Fisioterapia em Movimento. 2012;25(4).

17. Harada T, Saito DN, Kashikura K-I, Sato T, Yonekura Y, Honda M, et al. Asymmetrical neural substrates of tactile discrimination in humans: a functional magnetic resonance imaging study. The Journal of neuroscience. 2004;24(34):7524-30.

18. Lima RCM. Efeitos da adição da restrição de tronco à terapia por contensão induzida modificada em ambiente domiciliar: um ensaio clínico aleatorizado. 2013.

19. Uswatte G, Taub E, Morris D, Vignolo M, McCulloch K. Reliability and validity of the upperextremity Motor Activity Log-14 for measuring real-world arm use. Stroke. 2005;36(11):2493-6. 20. Wolf SL, Catlin PA, Ellis M, Archer AL, Morgan B, Piacentino A. Assessing Wolf motor function test as outcome measure for research in patients after stroke. Stroke. 2001;32(7):16359.

21. Wolf SL, Lecraw DE, Barton LA, Jann BB. Forced use of hemiplegic upper extremities to reverse the effect of learned nonuse among chronic stroke and head-injured patients. Experimental neurology. 1989;104(2):125-32.

22. Gosser SM, Rice MS. Efficiency of unimanual and bimanual reach in persons with and without stroke. Topics in stroke rehabilitation. 2015;22(1):56-62.

23. Messier S, Bourbonnais D, Desrosiers J, Roy Y. Kinematic analysis of upper limbs and trunk movement during bilateral movement after stroke. Archives of physical medicine and rehabilitation. 2006;87(11):1463-70.

24. Stock R, Thrane G, Askim T, Karlsen G, Langørgen E, Erichsen A, et al. Norwegian Constraint-Induced Therapy Multisite Trial: Adherence to Treatment Protocol Applied Early After Stroke. Journal of rehabilitation medicine. 2015;47(9):816-23.

25. Taub E, Uswatte G, Mark VW, Morris DM, Bishop-McKay S. Method for Enhancing RealWorld Use of a More Affected Arm in Chronic Stroke. 2013.

26. Morris D, Taub E, Mark V. Constraint-induced movement therapy: characterizing the intervention protocol. Europa medicophysica. 2006;42(3):257.

Citation: SILVA NT, YONOBI JH, ERAS-GARCIA R, GOMES JL, OLIVEIRA CB, MATUTI Influence of manual dominace on the unimanual and bimanual activities of post-stroke patients treated with constraint-induced therapy. BJMB. 2017: 9(1): $1-10$.

Editor: Guilherme Menezes Lage, Universidade Federal de Minas Gerais, MG, Brazil.

Copyright: @ 2017 SILVA NT, YONOBI JH, ERAS-GARCIA R, GOMES JL, OLIVEIRA CB, MATUTI and BJMB. This is an open-access article distributed under the terms of the Creative Commons Attribution-NonCommercial-

NoDerivatives 4.0 International License which permits unrestricted use, distribution, and reproduction in any medium, provided the original author and source are credited.

Funding: There was no funding for this study.

Competing interests: The authors have declared that no competing interests exist.

Download: http://socibracom.com/bjmb/index.php/bjmb/issue/view/38 